\title{
Spectrum of SU(2) gauge theory at large number of flavors
}

\author{
Jarno Rantaharju $\odot,{ }^{*}$ Tobias Rindlisbacher $\odot,{ }^{\dagger}$ Kari Rummukainen $\odot,{ }^{\ddagger}$ Ahmed Salami $\odot,{ }^{\S}$ and Kimmo Tuominen $\odot^{\|}$ \\ Department of Physics \& Helsinki Institute of Physics, University of Helsinki, \\ P.O. Box 64, FI-00014 University of Helsinki, Finland
}

(Received 16 September 2021; accepted 9 November 2021; published 10 December 2021)

\begin{abstract}
We present a numerical study of the spectrum of an asymptotically nonfree $\mathrm{SU}(2)$ gauge theory with $N_{f}=24$ massive fermion flavors. For such a large number of flavors, asymptotic freedom is lost and the massless theory is governed by a Gaussian fixed point at long distances. If fermions are massive they decouple at low energy scales and the theory is confining. We present a scaling law for the masses of the hadrons, glueballs and string tension as functions of fermion mass. The hadrons become effectively heavy quark systems, with masses approximately twice the fermion mass, whereas the energy scale of the confinement, probed by e.g., the string tension, is much smaller and vanishes asymptotically as $m_{\text {fermion }}^{2.18}$. Our results from lattice simulations are compatible with this behavior.
\end{abstract}

DOI: 10.1103/PhysRevD.104.114504

\section{INTRODUCTION}

Asymptotically free non-Abelian gauge-fermion theories are a cornerstone of our theoretical understanding of the elementary particle interactions of ordinary matter. The first principle methodologies of lattice field theory to solve these theories where perturbative methods are inapplicable are well established. Recently much effort has been devoted to studies of gauge-fermion theories whose matter content facilitates the existence of an infrared fixed point [1] as such theories are of interest for beyond-Standard-Model phenomenology [2-4]. On the lattice the properties of these types of theory have been studied for SU(2) gauge theory with matter fields in the fundamental [5-9] or adjoint [10-18] representation. For related studies in the SU(3) case, see e.g., [19-28] for fundamental representation fermions and [29-33] for two-index symmetric representation. Analyses have been extended also to the SU(4) gauge group with fermions in fundamental and higher representations [34,35].

However, less is known of the precise dynamics of gauge theories with many fermion flavors so that the theory is no longer asymptotically free. While such a particle content is not directly relevant for the Standard Model of elementary

\footnotetext{
jarno.rantaharju@helsinki.fi

tobias.rindlisbacher@helsinki.fi

kari.rummukainen@helsinki.fi

§ahmed.salami@helsinki.fi

"kimmo.i.tuominen@helsinki.fi
}

Published by the American Physical Society under the terms of the Creative Commons Attribution 4.0 International license. Further distribution of this work must maintain attribution to the author(s) and the published article's title, journal citation, and DOI. Funded by SCOAP . particle interactions, it poses an interesting challenge for the general understanding of gauge theory dynamics on one hand and on the development of computational methods on the other.

Broadly, the nonasymptotically free theories can be categorized as trivial or asymptotically safe. The former means that the theory develops an ultraviolet (UV) cutoff and can be interpreted consistently as a fundamental theory only as a free theory at short distances, while the latter means that a nonperturbative UV fixed point emerges and controls the short distance behavior of the theory.

In an earlier study [36], we analyzed this question in SU(2) gauge theory with 24 and 48 massless Dirac fermions by measuring the evolution of the coupling constant. Our results suggested that $\mathrm{SU}(2)$ gauge theory at these large numbers of fermions does not have an UV fixed point but is in the category of trivial theories. The results on the running coupling were seen to match well with the perturbative running in the infrared (IR).

In this paper we continue the lattice investigation of SU(2) gauge theory with 24 flavors. The aim is to complement our earlier results on the measurement of the coupling by a computation of the spectrum of the theory, i.e., the determination of long distance or IR behavior, as the quark ${ }^{1}$ mass $m_{q}$ is varied. When the quark mass is nonvanishing the infrared behavior of the theory changes: at energy scales smaller than the quark mass $m_{q}$, the quarks decouple and the physics is effectively that of confining $\mathrm{SU}(2)$ gauge theory with heavy quarks. The spectrum of the theory includes two-quark baryons, quark-antiquark mesons and glueballs, and the string tension is nonvanishing. In terms

\footnotetext{
${ }^{1}$ For concreteness, we call the fermions "quarks."
} 
of the renormalization group flow, the point $\left(g^{2}=0, m_{q}=0\right)$ is an infrared fixed point, and $g^{2}$ and $m_{q}$ are irrelevant and relevant parameters, respectively.

We introduce scaling laws for the hadron masses and quantities probing confinement (string tension, glueballs) as the quark mass is reduced. Our main result is that the hadrons behave as heavy quark systems, with masses close to $2 m_{q}$, whereas the energy scale characterizing confinement is much smaller, and asymptotically vanishes proportional to $m_{q}^{2.18}$. Our lattice measurements agree with these predictions, although the confinement scale turns out to be so small that we can only give upper limits to it. Together with our earlier work, the present paper establishes a consistent picture for the nonperturbative behaviors in this theory from IR to UV scales.

The paper is organized as follows: in Sec. II we introduce the expected scaling of the relevant mass scales of the theory. In Sec. III we briefly describe the lattice formulation and in Sec. IV we describe the lattice measurements and the results. In Sec. V we conclude and outline possibilities for further work.

\section{EXPECTED MASS SPECTRUM}

The solution of the 1-loop $\beta$-function for $\mathrm{SU}(2)$ gauge theory with $N_{f}$ massless quarks is

$$
g^{2}\left(\mu, N_{f}\right)=\frac{1}{2 \beta_{0}^{\left(N_{f}\right)} \log (\mu / \Lambda)} .
$$

Let us consider the case $N_{f}=24$. Now $\Lambda=\Lambda_{\mathrm{UV}}$ is the UV Landau pole, $\mu<\Lambda_{\mathrm{UV}}$ and $\beta_{0}^{(24)} \approx-0.0549$. Thus, when the energy scale $\mu \rightarrow 0$ the coupling constant $g^{2}$ vanishes, and the system is free in the infrared: there are no bound states, and the string tension vanishes.

However, if the quarks are massive the situation changes dramatically: when $\mu \ll m_{q}$, the quarks decouple and the system effectively becomes confining pure gauge SU(2) theory. String tension is nonvanishing, and the mass spectrum includes glueballs, quark-antiquark mesons and two-quark baryons.

The energy scale of the confinement of SU(2) gauge theory sets the mass scale of glueballs and string tension. We can make a rough estimate of the confinement scale with the 1-loop running of the coupling in $N_{f}=24$ and $N_{f}=0$ theories, and setting the couplings equal at $\mu=m_{q}$ :

$$
g^{2}\left(\mu=m_{q}, N_{f}=24\right)=g^{2}\left(\mu=m_{q}, N_{f}=0\right) .
$$

Let us call the $\Lambda$-parameter of the $N_{f}=0$ theory $\Lambda_{\mathrm{IR}}$. It is analogous to " $\Lambda_{\mathrm{QCD}}$ " of the pure gauge theory and is a proxy for the confinement energy scale. Alternatively, we could use a fixed large value of $g^{2}(\mu)$ to set the scale, and conclusions would remain unchanged.
Solving for $\Lambda_{\mathrm{IR}}$ in terms of the quark mass and the UV scale $\Lambda_{\mathrm{UV}}$, we obtain

$$
\frac{\Lambda_{\mathrm{IR}}}{\Lambda_{\mathrm{UV}}}=\left(\frac{m_{q}}{\Lambda_{\mathrm{UV}}}\right)^{1-\beta_{0}^{(24)} / \beta_{0}^{(0)}} \approx\left(\frac{m_{q}}{\Lambda_{\mathrm{UV}}}\right)^{2.18} .
$$

Thus, the confinement scale, and hence the glueball masses and the square root of the string tension, are proportional to $m_{q}^{2.18}$ at small quark masses. While the approximation (3) is based on 1-loop running and an abrupt mass threshold, it should become accurate in the limit $m_{q} / \Lambda_{\mathrm{UV}} \rightarrow 0$ because the coupling near $\mu=m_{q}$ will be small, and the small coupling region dominates the evolution of the scale.

On the other hand, because $m_{q} / \Lambda_{\mathrm{IR}} \gtrsim 1$ and grows as $m_{q}$ decreases, the two-quark hadrons are effectively "heavy quark" systems, with masses

$$
m_{\text {Hadron }} \approx 2 m_{q} \text {. }
$$

The size of the heavy quark system is proportional to its "Bohr radius," $\left[m_{q} g^{2}\left(m_{q}\right)\right]^{-1}$.

A more accurate estimation of the confinement scale can be obtained with the massive 2-loop $\beta$-function [37]. In this case the mass threshold is automatically taken care of by the $\beta$ - and $\gamma$-functions:

$$
\begin{aligned}
\mu \frac{d g^{2}}{d \mu} & =\beta\left(g^{2}, m_{q} / \mu\right), \\
\frac{\mu}{m_{q}} \frac{d m_{q}}{d \mu} & =-\gamma\left(g^{2}, m_{q} / \mu\right) .
\end{aligned}
$$

Because the quark mass is also running, we set the physical quark mass at the initial scale $\mu_{0}=2 m_{q, 0}$, give a range of initial values to $g^{2}\left(\mu_{0}\right)$ and evolve the equations to UV and IR until the coupling diverges. The result $\Lambda_{\mathrm{IR}} / \Lambda_{\mathrm{UV}}$ is shown in Fig. 1 as functions of $m_{q, 0} / \Lambda_{\mathrm{UV}}$. We observe that this result agrees with the approximation (3) at small $m_{q}$, but deviates from it substantially at larger $m_{q}$ when the mass threshold effects and higher order corrections affect the result significantly. Our goal is to observe how well this behavior is reproduced on the lattice.

\section{LATTICE FORMULATION}

On the lattice the theory is defined by the action

$$
S=S_{G}(U)+S_{F}(V)+c_{\mathrm{SW}} S_{\mathrm{SW}}(V),
$$

where $U$ is a $\mathrm{SU}(2)$ gauge link matrix in the fundamental representation, $V$ is a corresponding smeared gauge link, defined by hypercubic truncated stout smearing (HEX smearing) [38], $S_{G}$ is the Wilson gauge action, and $S_{F}$ and $S_{\mathrm{SW}}$ are, respectively, the Wilson fermion action and the clover term. The parameters of the action are the bare 


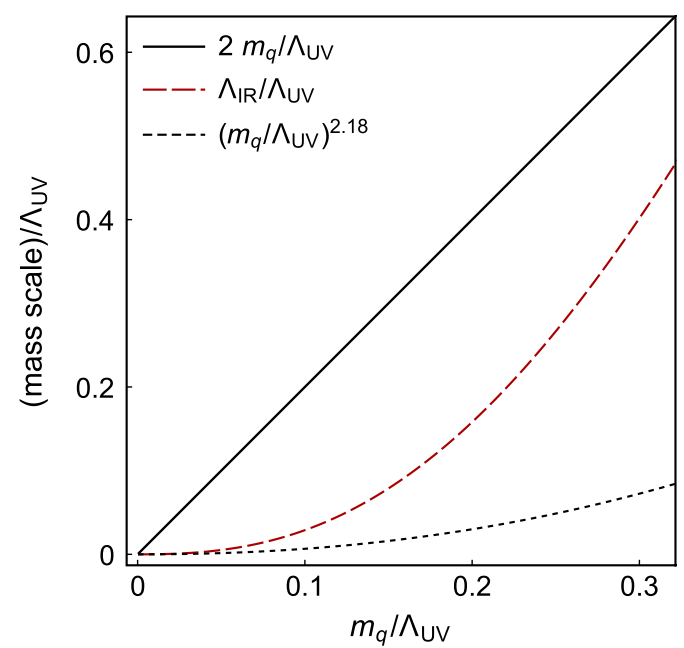

FIG. 1. The expected scaling of meson/baryon masses $m_{\text {Hadron }} \approx 2 m_{q}$ and the confinement scale $\Lambda_{\mathrm{IR}}$, which is proportional to the glueball masses and square root of the string tension, as functions of quark mass $m_{q}$. The long-dashed red line is obtained using the 2-loop massive quark $\beta$-function (5b) and the short-dashed black line is the approximation (3).

lattice gauge coupling $\beta_{L} \equiv 4 / g_{0}^{2}$, appearing in $S_{G}$, the "hopping parameter" $\kappa$ in $S_{F}$, and the SheikholeslamiWohlert coefficient $c_{\mathrm{SW}}$. We set $c_{S W}=1$, as is often used for HEX smeared fermions [17].

We simulate $N_{f}=24$ massive but mass-degenerate Wilson flavors on hypercubic, toroidal lattices of sizes $V=N_{s}^{3} \times N_{t}$, where $N_{s}$ and $N_{t}$ refer to the number of lattice sites in spatial and temporal direction. These cover the values $N_{t} \in\{32,40,48\}$ and $N_{s} \in\left\{N_{t} / 2,3 N_{t} / 4, N_{t}\right\}$. The boundary conditions for the gauge field are periodic in all directions, whereas for the fermion fields, they are periodic only in spatial but antiperiodic in temporal direction, as usual.

Simulations are carried out using a hybrid Monte Carlo (HMC) algorithm with a leapfrog integrator and chronological initial values for the fermion matrix inversion [39]. The HMC trajectories have unit-length and the number of leapfrog steps is tuned to yield acceptance rates above $80 \%$ (with a few exceptions).

The physical quark mass is determined by the lattice PCAC [40] relation

$$
\operatorname{am}_{q}\left(x_{4}\right)=\frac{\left(\partial_{4}^{*}+\partial_{4}\right) f_{A}\left(x_{4}\right)}{4 f_{P}\left(x_{4}\right)},
$$

where $\partial_{4}$ and $\partial_{4}^{*}$ are, respectively, forward and backward lattice time-derivative operators, and

$$
\begin{aligned}
& f_{A}\left(x_{4}\right)=-\frac{1}{N_{s}^{3} N_{t}} \sum_{\mathbf{x}, y, a}\left\langle A_{\mu}^{a}\left(\mathbf{y}, y_{4}\right) A_{\mu}^{a}\left(\mathbf{x}, x_{4}+y_{4}\right)\right\rangle, \\
& f_{P}\left(x_{4}\right)=\frac{1}{N_{s}^{3} N_{t}} \sum_{\mathbf{x}, y, a}\left\langle P^{a}\left(\mathbf{y}, y_{4}\right) P^{a}\left(\mathbf{x}, x_{4}+y_{4}\right)\right\rangle
\end{aligned}
$$

are the axial current and pseudoscalar density correlation functions with point sources:

$$
\begin{aligned}
& A_{\mu}^{a}(x)=\bar{\psi}(x) \gamma_{\mu} \gamma_{5} \frac{1}{2} \tau^{a} \psi(x), \\
& P^{a}(x)=\bar{\psi}(x) \gamma_{5} \frac{1}{2} \tau^{a} \psi(x) .
\end{aligned}
$$

For each value of the bare gauge coupling $\beta_{L} \equiv 4 / g_{0}^{2}$, the hopping parameter $\kappa$ in the Wilson fermion action is tuned to cover a range of PCAC fermion masses for which we study the behavior of the spectrum from the chiral limit to heavy quarks. The masses of color singlet meson states are then determined by fitting the time sliced average correlation functions with Coulomb gauge fixed wall sources:

$$
{ }^{(w)} \Gamma^{a}\left(x_{4}\right)=\sum_{\mathbf{x}, \mathbf{y}} \bar{\psi}\left(\mathbf{x}, x_{4}\right) \Gamma \frac{1}{2} \tau^{a} \psi\left(\mathbf{y}, x_{4}\right),
$$

with $\Gamma$ representing an element of the Dirac algebra.

\section{RESULTS}

Let us now turn to the details of the simulations and the results we have obtained on the spectrum of physical states. The simulation parameters and corresponding PCAC quark masses, pseudoscalar (" $\pi$ ") and vector (" $\rho$ ") meson masses, together with the acceptance rates and accumulated statistics, are given in the Appendix in Tables II-IX.

\section{A. Phase diagram}

For the bare gauge coupling we use values $\beta_{L}=4 / g_{0}^{2} \in\{-0.25,0.001,0.25\}$. The small values of $\beta_{L}$ might at first seem to imply that the gauge field is deep in an unphysical strong coupling "bulk phase." This is in general, however, not the case, as the Wilson fermions induce an effective positive shift in $\beta_{L}[41,42]$. This is to leading order proportional to the number of flavors and therefore substantial for $N_{f}=24$. Indeed, the above values of $\beta_{L}$ were successfully used in the measurement of the coupling constant evolution at vanishing fermion masses [36]. A similar effect has been reported in [43] for staggered fermions.

The value of the $\beta_{L}$ affects the lattice spacing, but to the opposite direction than in lattice QCD: the lattice spacing is made smaller when $\beta_{L}$ is decreased. Because of the Landau pole the theory on the lattice does not have a continuum limit. Nevertheless, we anticipate that the theory has a scaling window where the physical results are independent of the actual cutoff scale.

In Fig. 2(a) we show the measured PCAC quark masses $m_{q}$ as functions of the hopping parameter $\kappa$. It is evident that the system has an abrupt phase transition into the unphysical "bulk phase" at small values of $\kappa$. The approximate location of the bulk transition is shown with vertical 

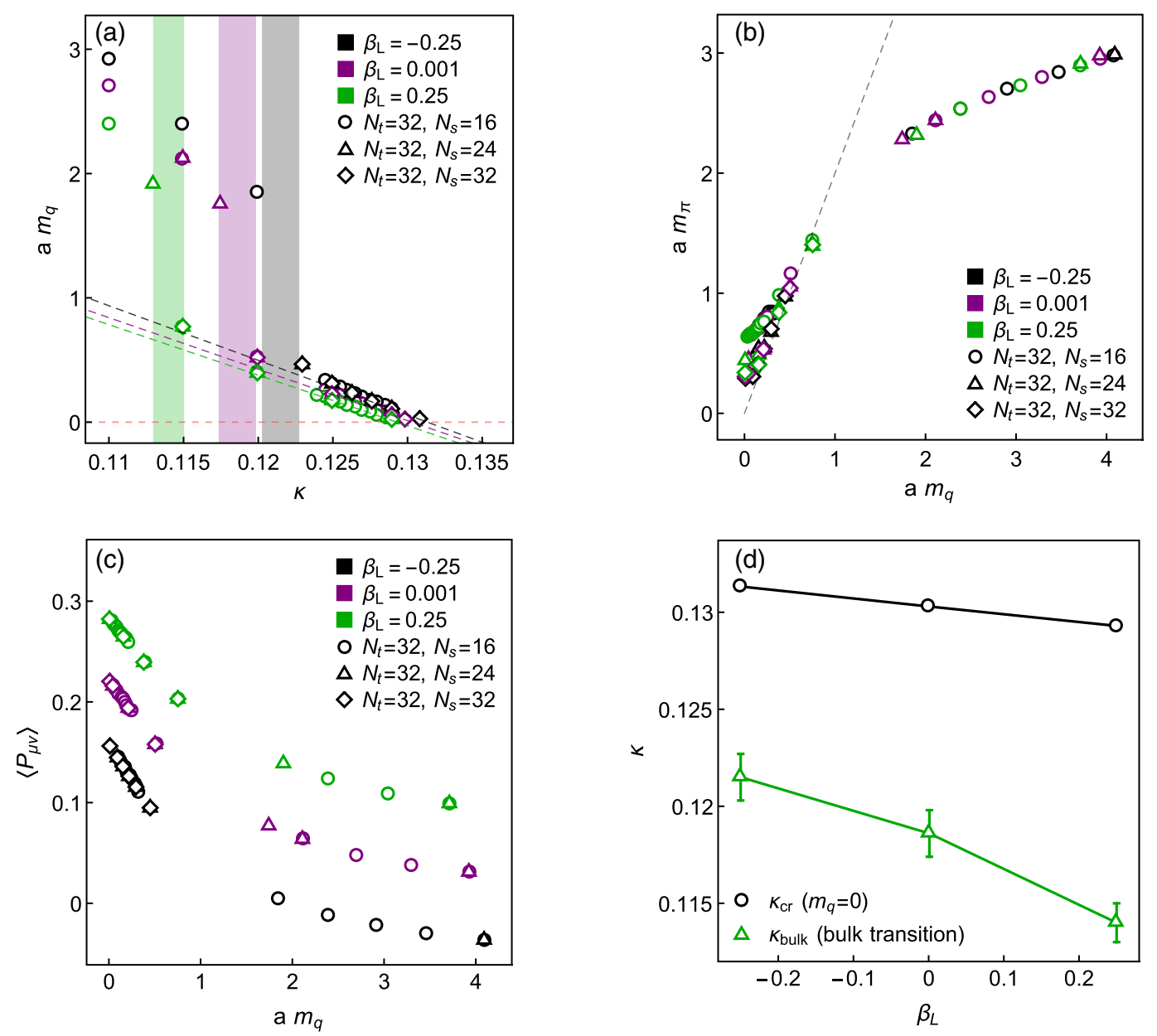

FIG. 2. (a) The PCAC quark masses $m_{q}$ as functions of $\kappa$, measured at $\beta_{L}=-0.25,0.001,0.25$ and lattice sizes $V=N_{s}^{3} \times N_{t}$ with $N_{t}=32, N_{s}=16,24,32$. Here, and in what follows, different colors indicate the $\beta_{L}$-values and a plot symbol shapes the volume. The vertical shaded bands show the approximate locations of the bulk phase transitions at different $\beta_{L}$. The dashed lines are linear fits to three smallest $m_{q}$-values for each $\beta_{L}$, used to determine the critical value $\kappa_{\mathrm{cr}}$. (b) The pseudoscalar meson ("pion") mass $m_{\pi}$. (c) Plaquette as functions of $m_{q}$. In (b) the dashed line is $m_{\pi}=2 m_{q}$-line. (d) The phase diagram of the system. The physical domain is between the bulk transition and critical $\left(m_{q}=0\right)$ lines.

shaded bands for each value of $\beta_{L}$. On the large- $\kappa$ side of the transition there is a range of $\kappa$-values where $m_{q}$ decreases from $0.8-0.9$ down to 0 as $\kappa$ is increased (here, and in what follows, dimensionful quantities are given in units of the lattice spacing $a$, unless specified otherwise). This is the range where physics can be extracted. The bulk transition is also visible in other observables, for example the pseudoscalar mass [Fig. 2(b)] and plaquette expectation value [Fig. 2(c)]. As expected, the pseudoscalar mass is close to $2 m_{q}$.

The $\left(\beta_{L}, \kappa\right)$-plane phase diagram is shown in Fig. 2(d). The physically relevant domain is between the bulk transition and the critical line $\kappa_{\mathrm{cr}}\left(\beta_{L}\right)$, where $m_{q}=0$. The values of the critical $\kappa_{\mathrm{cr}}\left(\beta_{L}\right)$ have been obtained by linear extrapolation of the measured $m_{q}(\kappa)$ at smallest quark masses. The numerical values of $\kappa_{\mathrm{cr}}$ and the bulk transition $\kappa_{\text {bulk }}$ are shown in Table I.

\section{B. Finite size effects}

As discussed in Sec. II, the size of the hadrons grow as $m_{q} \rightarrow 0$, and we can expect large finite size effects in this limit. This is evident in Fig. 3(a), where we show the pseudoscalar "pion" masses $m_{\pi}$ measured from volumes $N_{s}^{3}$ from $12^{3}$ up to $32^{3}$ at different values of $\beta_{L}$. At small

TABLE I. The table lists for a system of size $V=N_{s}^{3} \times N_{t}$ with $N_{s}=N_{t}=32$ and different $\beta$-values; the values of $\kappa_{\text {cr }}$ and $\kappa_{\text {bulk }}$, being, respectively, the values of $\kappa$ for which the PCAC quark mass $m_{q}$ vanishes, and at which the bulk transition occurs.

\begin{tabular}{ccccc}
\hline \hline$N_{t}$ & $N_{s}$ & $\beta_{L}$ & $\kappa_{\text {cr }}$ & $\kappa_{\text {bulk }}$ \\
\hline 32 & 32 & -0.25 & $0.131327(4)$ & $0.1215(12)$ \\
32 & 32 & 0.001 & $0.130296(2)$ & $0.1186(12)$ \\
32 & 32 & 0.25 & $0.129297(2)$ & $0.1140(10)$ \\
\hline \hline
\end{tabular}



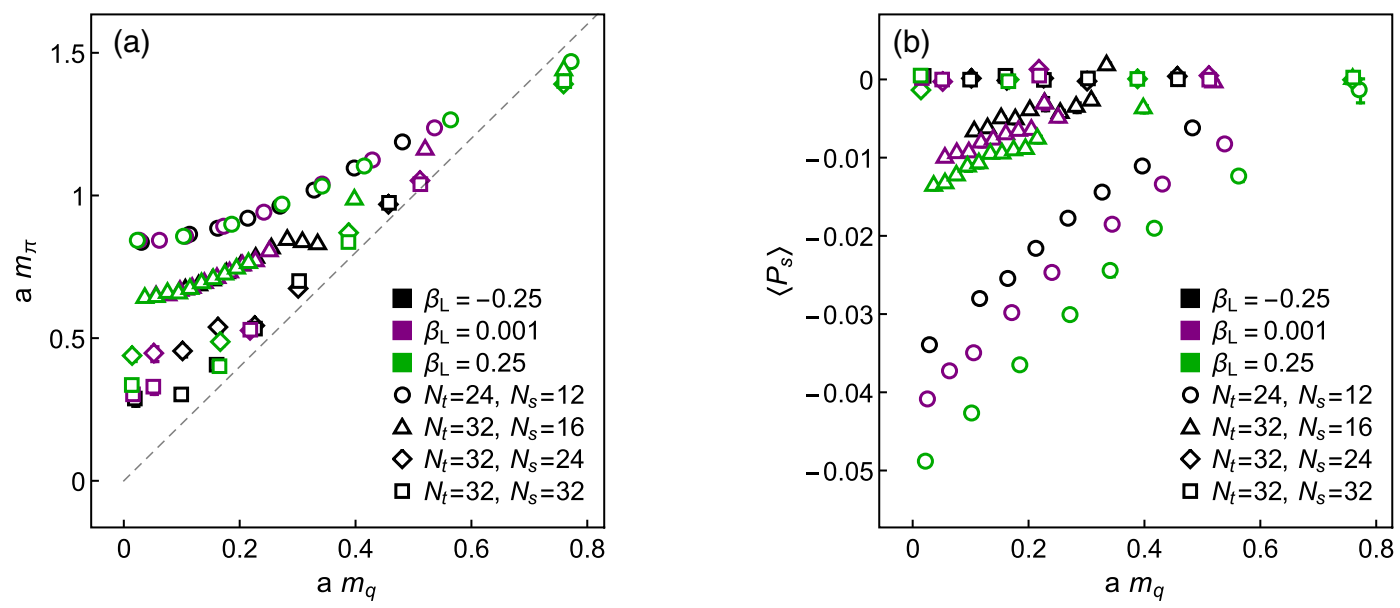

FIG. 3. The figure shows for three different values of $\beta_{L}=-0.25,0.001,0.25$ and lattice sizes $V=N_{s}^{3} \times N_{t}$ with $N_{s}=16,24,32$ at $N_{t}=32$ and $N_{s}=12$ at $N_{t}=24$ (a) the pion mass $m_{\pi}$ and (b) the expectation value of spatial Polyakov loop $\left\langle P_{s}\right\rangle$ as functions of the PCAC quark mass $m_{q}$. Note that colors (black, purple, green) are used to distinguish between different values of $\beta_{L}$ and symbols (circle, triangle, diamond) are used to distinguish the different system sizes. For comparison, the black, dashed, diagonal line in (a) shows the free quark case, $m_{\pi}=2 m_{q}$.

volumes the mass plateaus roughly at $m_{\pi} \approx 1 /\left(N_{s} a\right)$ as $m_{q}$ is lowered. Interestingly, there is very little dependence on the value of the lattice gauge coupling $\beta_{L}$, indicating that it is the quark mass which is important for finite size effects.

In Fig. 3(b) we show the expectation values of the spatial Polyakov loop $\langle P\rangle$ as functions of the quark mass. A nonzero value indicates that the system becomes spatially deconfined, i.e., the volume is too small to contain the confinement physics. The negative expectation value is due to the periodic boundary conditions for fermions to spatial directions. Clearly, at small volumes the magnitude of the expectation values of the Polyakov lines grow as the quark mass is decreased. The magnitude of $\langle P\rangle$ is expected to decrease exponentially with the length of the line, $N_{s}$. Nevertheless, the behavior changes qualitatively as the volume is increased: $\langle P\rangle$ remains zero down to progressively smaller values of $m_{q}$. At $N_{s}=32,\langle P\rangle$ remains zero at all $m_{q}$, within our statistical accuracy.

\section{Meson spectrum}

The spectrum of pseudoscalar and vector mesons is shown in detail in Fig. 4 for the range of $m_{q}$ away from the bulk phase as discussed above. The pion masses as a function of the quark mass are shown in panels (a) and (c), and the vector meson masses in panels (b) and (d). The results corresponding to $\beta_{L}$-values, $-0.25,0.001$ and 0.25 , are shown and different values are indicated by colors black, blue and red respectively. In (a) and (b) the results are shown for system sizes $N_{t}=N_{s}=L$ with $L=32$ (circles), $L=40$ triangles and $L=48$ (diamonds), while in (c) and (d) the results are shown for $N_{t}=48$ with $N_{s}=24$ (circles), $N_{s}=36$ (triangles) and $N_{s}=48$ (diamonds).
Both the quark mass $m_{q}$ and the hadron masses are in units of the inverse lattice spacing.

As expected, the hadron masses very closely follow the $2 m_{q}$-line, slightly above it, and significant deviations appear only when $m_{\text {hadron }} \lesssim 1 / L$, the inverse spatial size of the lattice. There is also no significant difference between pseudoscalar and vector mesons within the accuracy of our measurements. This demonstrates the heavy quark nature of the mesons.

The results are independent of the bare lattice coupling $\beta_{L}$ in the sense that the results fall on a universal line on the $\left(m_{q}, m_{\text {Hadron }}\right)$-plane. We remind the reader that the lattice spacing becomes smaller as $\beta_{L}$ is decreased (bare coupling grows when lattice spacing is decreased), but the theory has no continuum limit because of the UV Landau pole. Nevertheless, the observed universal behavior in $\beta_{L}$ indicates that the theory has a scaling window where the physical results remain independent of the cutoff scale.

\section{String tension and glueballs}

In Sec. II it was predicted that the confinement scale, which determines the string tension and the glueball masses, scales as $\left(m_{q} / \Lambda_{\mathrm{UV}}\right)^{2.18}$ at small $m_{q}$, with deviations expected at larger $m_{q}$ (see Fig. 1). We measure the string tension $\sigma$ by constructing Wilson loops using spatially smeared gauge fields, with five different APE smearing levels [44] with up to 32 smearings [45]. We measure Wilson loops $W(\mathbf{r}, t)$, where $\mathbf{r}$ is an integer multiple of one of the spatial vectors $(1,0,0),(1,1,0)$, $(1,1,1),(2,1,0),(2,1,1)+$ reflections and permutations. The large- $t$ behavior of the Wilson loops is fitted with an effective potential 

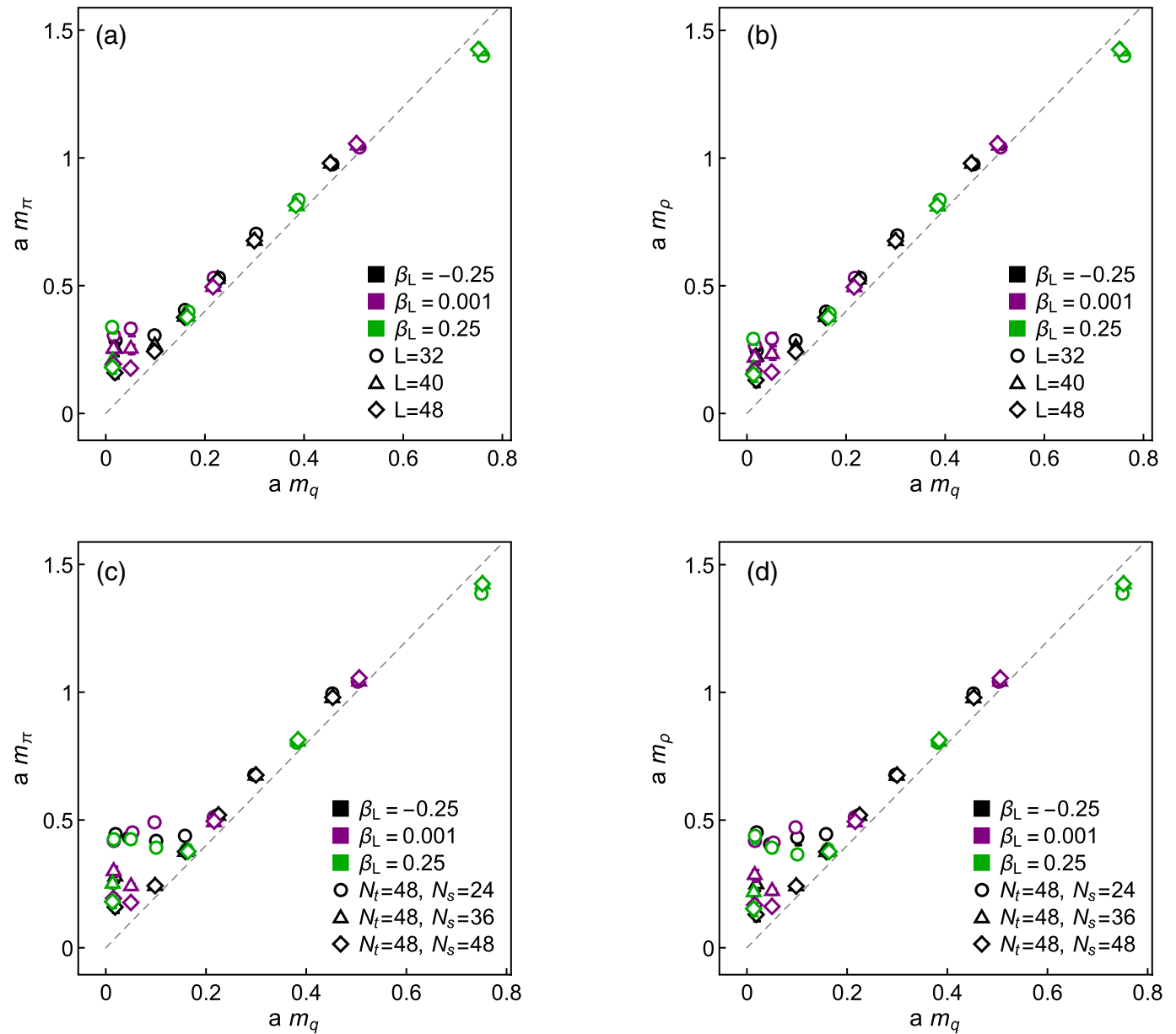

FIG. 4. The pseudoscalar meson mass $m_{\pi}(\mathrm{a}, \mathrm{c})$ and the vector meson mass $m_{\rho}(\mathrm{b}, \mathrm{d})$ as functions of the PCAC quark mass $m_{q}$ for three values of $\beta_{L}=-0.25,0.001,0.25$ and system sizes $V=N_{s}^{3} \times N_{t}$, where in (a, b) $N_{t}=N_{s}=L, L=32,40,48$, and in (c, d) $N_{t}=48$, $N_{s}=24,36,48$. The colors (black, purple, green) are used to distinguish between different values of $\beta_{L}$ and symbols (circle, triangle, diamond) are used to distinguish the different system sizes. For comparison, the black, dashed, diagonal lines show the pion mass in the case of free quarks, $m_{\pi}=2 m_{q}$.

$$
\log \frac{W(\mathbf{r}, t+1)}{W(\mathbf{r}, t)}=\frac{A}{r}+\sigma r+B,
$$

where $r=|\mathbf{r}|$ and $A, B$ and $\sigma$ are fit parameters.

However, it turns out that the string tension is too small to be reliably measured using our lattice sizes and statistics. We can only present upper limits for the string tension, measured from the largest $t$-distance before the statistical errors make the measurement meaningless. Nevertheless, the measurements have not yet stabilized as $t$ is changed. This is shown in Fig. 5. We also attempted to do a generalized eigenvalue analysis of the Wilson loops between different smearing levels, but this did not stabilize either, presumably because the spacing between eigenvalues is too small.

The upper limits of the square root of the string tension are shown in Fig. 6. To guide the expectation, the simple scaling ansatz (3) is shown by the dashed line, with arbitrary scaling.
Finally, we attempt to measure glueball correlation functions using spatially smeared gauge links, with up to 100 APE smearing steps. From the smeared gauge fields we construct operators coupling to scalar $J^{P C}=0^{++}$and tensor $2^{++}$glueballs. On the lattice the operators transform under cubic group representations [46,47], and we measure operators under six different representations. Considering the difficulties in measuring the string tension, it is perhaps not surprising that we were not able to reliably measure glueball masses. The correlation functions coupling to the $\mathrm{O}^{++}$state are very noisy due to the disconnected contribution. Correlation functions of operators in the cubic group representations $E^{++}$and $T_{2}^{++}$, which couple to the continuum $2^{++}$state, do not have a disconnected part and are somewhat better behaved. Nevertheless, we were not able to obtain the asymptotic state in these channels.

We assign the failure of the glueball mass measurement to the (expected) small value of the masses. This implies that the numerous excited states coupling to the same 


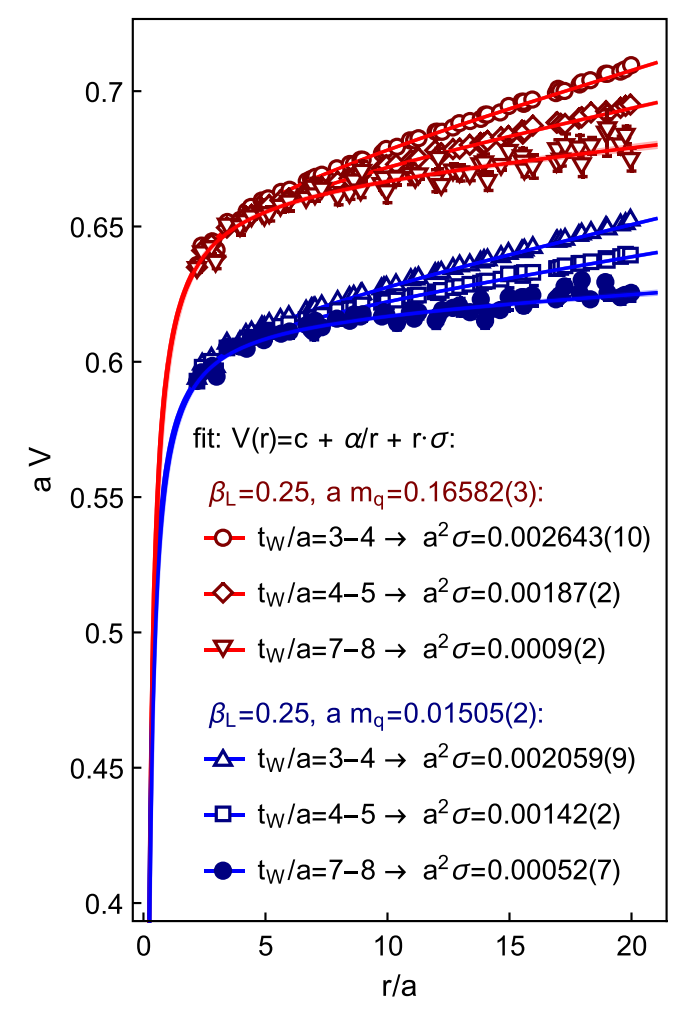

FIG. 5. Examples of fitting the effective potential while the $t$-distance of the Wilson loops, $t_{w}$, is varied. The result does not settle to an asymptotic value, and the result is only an upper bound.

operators also have small masses, and it becomes very difficult to find the ground state. We attempted to use generalized eigenvalue analysis of operators with different numbers of smearing steps, but the small mass gaps between states rendered the procedure unstable.

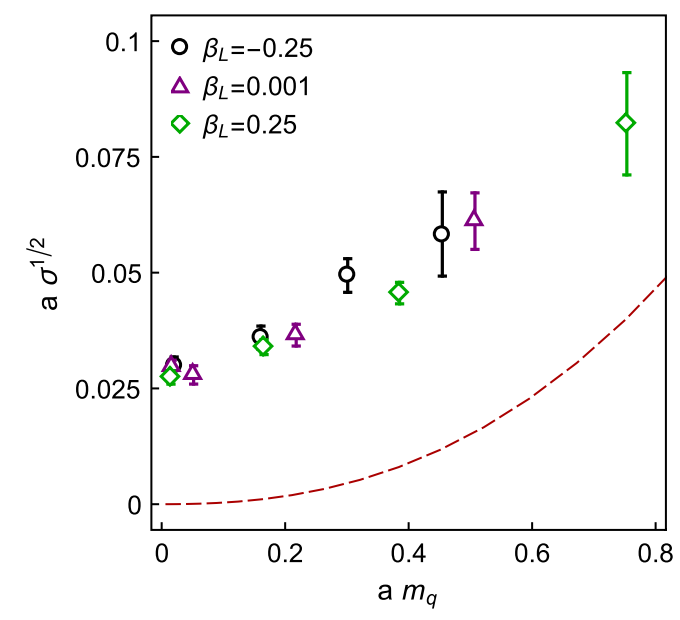

FIG. 6. The upper limit of the square root of the string tension $\sigma^{1 / 2}$ as function of the quark mass $a m_{q}$. The dashed line shows the confinement scale $\Lambda_{\mathrm{IR}}$ from Fig. 1, assuming (arbitrarily) that $a \Lambda_{\mathrm{UV}} \sim 24$.

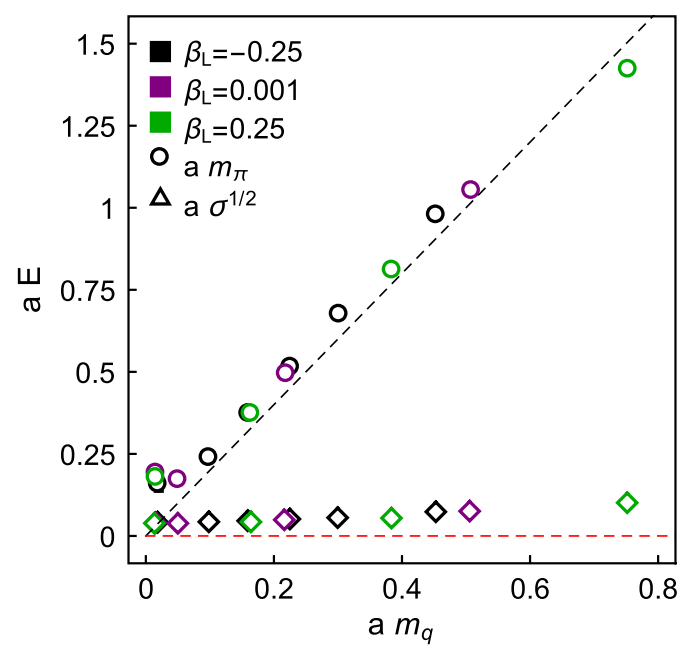

FIG. 7. The pseudoscalar mass $m_{\pi}$ and the upper limit of the square root of the string tension $\sigma^{1 / 2}$ as functions of $m_{q}$. The data is measured from lattices of size $48^{4}$. The diagonal, black, dashed line corresponds to $E=2 m_{q}$. The vertical, red, dashed line indicates $E=0$.

In Fig. 7 we plot together the pseudoscalar mass and the upper limit of the square root of the string tension, measured from $48^{4}$ lattices. $m_{\pi}$ is more than an order of magnitude larger than the upper limit of $\sigma^{1 / 2}$, and at the smaller end of the $m_{\pi}$-range the true hierarchy is expected to become much larger. Compared with Fig. 1, we can conclude that the physics on our lattices correspond to the case where quark masses are substantially below the Landau pole, $m_{q} / \Lambda_{\mathrm{UV}} \ll 1$. Because in the lattice units $m_{q} a \lesssim 1$ this implies that the Landau pole is effectively at considerably larger scale than the inverse lattice spacing, $\Lambda_{\mathrm{UV}} \gg 1 / a$. This was also observed in our earlier study of the running coupling at massless theory [36].

It is difficult to reach larger values of $m_{q} / \Lambda_{\mathrm{UV}}$ with lattice simulations; trying to make the bare coupling stronger and the quarks heavier moves us towards the direction of strong lattice artifacts. With our choice of the lattice action the bulk transition prevents us from using heavier quarks.

\section{CONCLUSIONS}

We have studied the mass spectrum of $\mathrm{SU}(2)$ gauge theory with $N_{f}=24$ Dirac fermions on the lattice, complementing our earlier analysis [36] on the running coupling in this theory. The massless theory is free in the infrared, without bound states. When the quark mass $m_{q}$ is nonzero the behavior is different: in the infrared the theory is confining, and its spectrum includes mesons, two-quark baryons and glueballs, and the string tension is nonvanishing. We have presented scaling relations for the hadron masses and for the confinement scale as functions of the quark mass. The hadrons behave effectively as heavy quark 
systems with masses close to $2 m_{q}$, whereas the confinement scale decreases faster, at small $m_{q}$ proportional to $m_{q}^{2.18}$.

The scaling relations imply that glueball masses and the square root of the string tension $\sigma^{1 / 2}$ are much smaller than hadron masses unless quarks are very heavy, with masses of the order of the Landau pole of the theory. The results from lattice simulations confirm this behavior: $\sigma^{1 / 2}$ is more than an order of magnitude smaller than the hadron masses. However, we were only able to give an upper limit for $\sigma^{1 / 2}$ in the range of quark masses studied, and we expect the true result to be substantially below the upper limit at small $m_{q}$.

To fully verify the behavior of the confinement scale as $m_{q}$ is varied we would need to use much heavier quarks and stronger lattice coupling, so that the quark mass should be close to the Landau pole, $m_{q} / \Lambda_{\mathrm{UV}} \lesssim 1$. However, lattice bulk transition and increased lattice artifacts prevent us from moving significantly to that direction. Furthermore, because the theory is only defined up to the UV cutoff scale, moving quark masses close to it makes the relevant physics sensitive to the details of the UV regularization used.

\section{ACKNOWLEDGMENTS}

The support of the Academy of Finland Grants No. 308791, No. 310130, No. 319066 and No. 320123 is acknowledged. The authors wish to acknowledge CSCIT Center for Science, Finland, for generous computational resources.

\section{APPENDIX: TABLES OF SIMULATION PARAMETERS AND RESULTS}

A summary of the simulation parameters and corresponding PCAC quark masses, pion and rho-meson masses, as well as the acceptance rates and accumulated statistics (after subtraction of nonthermalized configurations), is given in Tables II-IX.

TABLE II. The table shows for systems of size $V=N_{s}^{3} \times N_{t}$ with $N_{t}=24$ and $N_{s}=12$ the simulated values of $\beta_{L}$ and $\kappa$, the corresponding PCAC quark mass, $m_{q}$, pion and $\rho$-meson masses, $m_{\pi}, m_{\rho}$, the acceptance rate of the HMC trajectories, and the number of usable configurations (after thermalization).

\begin{tabular}{|c|c|c|c|c|c|c|c|c|}
\hline$N_{t}$ & $N_{s}$ & $\beta_{L}$ & $\kappa$ & $m_{q}$ & $m_{\pi}$ & $m_{\rho}$ & acceptance rate & statistics \\
\hline 24 & 12 & -0.25 & 0.1309 & $0.037(2)$ & $0.84(5)$ & $0.91(5)$ & 0.98 & $8.8 \mathrm{k}$ \\
\hline 24 & 12 & -0.25 & 0.129 & $0.133(5)$ & $0.79(5)$ & $0.80(7)$ & 0.98 & $9 \mathrm{k}$ \\
\hline 24 & 12 & -0.25 & 0.128 & $0.194(5)$ & $0.86(3)$ & $0.87(4)$ & 0.99 & $9.3 \mathrm{k}$ \\
\hline 24 & 12 & -0.25 & 0.127 & $0.263(5)$ & $0.91(3)$ & $0.93(3)$ & 0.99 & $9.5 \mathrm{k}$ \\
\hline 24 & 12 & -0.25 & 0.126 & $0.337(8)$ & $0.98(4)$ & $0.99(4)$ & 0.99 & $9.7 \mathrm{k}$ \\
\hline 24 & 12 & -0.25 & 0.125 & $0.40(2)$ & $1.00(4)$ & $1.00(5)$ & 0.99 & $9.7 \mathrm{k}$ \\
\hline 24 & 12 & -0.25 & 0.124 & $0.49(2)$ & $1.08(3)$ & $1.09(4)$ & 0.99 & $9.5 \mathrm{k}$ \\
\hline 24 & 12 & -0.25 & 0.123 & $0.58(2)$ & $1.13(3)$ & $1.14(3)$ & 0.99 & $9.8 \mathrm{k}$ \\
\hline 24 & 12 & 0.001 & 0.1299 & $0.029(1)$ & $0.77(4)$ & $0.76(6)$ & 0.99 & $9.2 \mathrm{k}$ \\
\hline 24 & 12 & 0.001 & 0.129 & $0.077(3)$ & $0.86(4)$ & $0.88(5)$ & 0.99 & $9.4 \mathrm{k}$ \\
\hline 24 & 12 & 0.001 & 0.128 & $0.129(3)$ & $0.85(3)$ & $0.84(4)$ & 0.99 & $9.5 \mathrm{k}$ \\
\hline 24 & 12 & 0.001 & 0.1265 & $0.202(6)$ & $0.85(4)$ & $0.87(4)$ & 0.99 & $9.5 \mathrm{k}$ \\
\hline 24 & 12 & 0.001 & 0.125 & $0.288(9)$ & $0.93(4)$ & $0.95(4)$ & 0.99 & $9.6 \mathrm{k}$ \\
\hline 24 & 12 & 0.001 & 0.123 & $0.411(8)$ & $1.03(3)$ & $1.03(3)$ & 0.99 & $10.2 \mathrm{k}$ \\
\hline 24 & 12 & 0.001 & 0.1215 & $0.50(2)$ & $1.06(3)$ & $1.07(3)$ & 0.99 & $10.1 \mathrm{k}$ \\
\hline 24 & 12 & 0.001 & 0.12 & $0.66(1)$ & $1.22(2)$ & $1.22(2)$ & 0.99 & $10.2 \mathrm{k}$ \\
\hline 24 & 12 & 0.25 & 0.129 & $0.0270(8)$ & $0.81(4)$ & $0.79(5)$ & 0.99 & $9.6 \mathrm{k}$ \\
\hline 24 & 12 & 0.25 & 0.127 & $0.121(3)$ & $0.82(3)$ & $0.85(4)$ & 0.99 & $9.9 \mathrm{k}$ \\
\hline 24 & 12 & 0.25 & 0.125 & $0.231(6)$ & $0.93(3)$ & $0.96(3)$ & 0.99 & $10.1 \mathrm{k}$ \\
\hline 24 & 12 & 0.25 & 0.123 & $0.33(1)$ & $0.98(4)$ & $0.99(4)$ & 0.99 & $10.6 \mathrm{k}$ \\
\hline 24 & 12 & 0.25 & 0.1215 & $0.392(9)$ & $0.98(4)$ & $1.00(4)$ & 0.99 & $10.5 \mathrm{k}$ \\
\hline 24 & 12 & 0.25 & 0.12 & $0.50(1)$ & $1.10(3)$ & $1.11(3)$ & 0.99 & $10.3 \mathrm{k}$ \\
\hline 24 & 12 & 0.25 & 0.1175 & $0.64(2)$ & $1.16(4)$ & $1.16(4)$ & 0.99 & $6 \mathrm{k}$ \\
\hline 24 & 12 & 0.25 & 0.115 & $0.92(2)$ & $1.41(2)$ & $1.41(2)$ & 0.99 & $11.5 \mathrm{k}$ \\
\hline
\end{tabular}


TABLE III. Same as Table II for $N_{t}=32, N_{s}=32$.

\begin{tabular}{|c|c|c|c|c|c|c|c|c|}
\hline$N_{t}$ & $N_{s}$ & $\beta_{L}$ & $\kappa$ & $m_{q}$ & $m_{\pi}$ & $m_{\rho}$ & acceptance rate & statistics \\
\hline 32 & 32 & -0.25 & 0.1309 & $0.0207(2)$ & $0.28(3)$ & $0.24(3)$ & 0.89 & $0.4 \mathrm{k}$ \\
\hline 32 & 32 & -0.25 & 0.129 & $0.1008(2)$ & $0.299(5)$ & $0.284(5)$ & 0.84 & $0.5 \mathrm{k}$ \\
\hline 32 & 32 & -0.25 & 0.1277 & $0.1619(2)$ & $0.403(6)$ & $0.397(6)$ & 0.84 & $1.2 \mathrm{k}$ \\
\hline 32 & 32 & -0.25 & 0.1263 & $0.2282(2)$ & $0.530(2)$ & $0.530(2)$ & 0.84 & $1.3 \mathrm{k}$ \\
\hline 32 & 32 & -0.25 & 0.125 & $0.3043(2)$ & $0.697(6)$ & $0.695(7)$ & 0.82 & $1.5 \mathrm{k}$ \\
\hline 32 & 32 & -0.25 & 0.123 & $0.4595(5)$ & $0.971(3)$ & $0.971(3)$ & 0.84 & $1.7 \mathrm{k}$ \\
\hline 32 & 32 & 0.001 & 0.1299 & $0.0176(1)$ & $0.30(2)$ & $0.26(2)$ & 0.89 & $1.4 \mathrm{k}$ \\
\hline 32 & 32 & 0.001 & 0.129 & $0.0526(1)$ & $0.33(3)$ & $0.29(3)$ & 0.83 & $0.9 \mathrm{k}$ \\
\hline 32 & 32 & 0.001 & 0.125 & $0.2201(2)$ & $0.53(2)$ & $0.52(2)$ & 0.83 & $1.4 \mathrm{k}$ \\
\hline 32 & 32 & 0.001 & 0.12 & $0.5136(5)$ & $1.036(2)$ & $1.036(2)$ & 0.85 & $1.7 \mathrm{k}$ \\
\hline 32 & 32 & 0.25 & 0.129 & $0.0155(1)$ & $0.33(2)$ & $0.29(2)$ & 0.86 & $0.7 \mathrm{k}$ \\
\hline 32 & 32 & 0.25 & 0.125 & $0.1669(1)$ & $0.398(2)$ & $0.391(2)$ & 0.89 & $1.1 \mathrm{k}$ \\
\hline 32 & 32 & 0.25 & 0.12 & $0.3894(2)$ & $0.833(5)$ & $0.832(5)$ & 0.88 & $1.5 \mathrm{k}$ \\
\hline 32 & 32 & 0.25 & 0.115 & $0.762(2)$ & $1.40(2)$ & $1.40(2)$ & 0.88 & $1.9 \mathrm{k}$ \\
\hline
\end{tabular}

TABLE IV. Same as Table II for $N_{t}=40, N_{s}=20$.

\begin{tabular}{|c|c|c|c|c|c|c|c|c|}
\hline$N_{t}$ & $N_{s}$ & $\beta_{L}$ & $\kappa$ & $m_{q}$ & $m_{\pi}$ & $m_{\rho}$ & acceptance rate & statistics \\
\hline 40 & 20 & -0.25 & 0.1309 & $0.0226(1)$ & $0.50(2)$ & $0.50(2)$ & 0.95 & $2 \mathrm{k}$ \\
\hline 40 & 20 & -0.25 & 0.1302 & $0.0509(1)$ & $0.48(2)$ & $0.51(2)$ & 0.97 & $1.3 \mathrm{k}$ \\
\hline 40 & 20 & -0.25 & 0.129 & $0.1045(2)$ & $0.56(1)$ & $0.54(2)$ & 0.86 & $1 \mathrm{k}$ \\
\hline 40 & 20 & -0.25 & 0.1277 & $0.1646(2)$ & $0.51(2)$ & $0.48(2)$ & 0.85 & $0.6 \mathrm{k}$ \\
\hline 40 & 20 & -0.25 & 0.1263 & $0.2292(3)$ & $0.64(2)$ & $0.65(2)$ & 0.88 & $0.3 \mathrm{k}$ \\
\hline 40 & 20 & -0.25 & 0.125 & $0.2978(5)$ & $0.663(3)$ & $0.663(3)$ & 0.89 & $0.2 \mathrm{k}$ \\
\hline 40 & 20 & -0.25 & 0.123 & $0.4538(4)$ & $0.971(4)$ & $0.972(4)$ & 0.86 & $2 \mathrm{k}$ \\
\hline 40 & 20 & 0.001 & 0.1299 & $0.0189(1)$ & $0.53(2)$ & $0.57(2)$ & 0.97 & $2 \mathrm{k}$ \\
\hline 40 & 20 & 0.001 & 0.129 & $0.0547(1)$ & $0.52(2)$ & $0.57(2)$ & 0.88 & $1 \mathrm{k}$ \\
\hline 40 & 20 & 0.001 & 0.1278 & $0.1019(1)$ & $0.56(2)$ & $0.54(2)$ & 0.96 & $1.4 \mathrm{k}$ \\
\hline 40 & 20 & 0.001 & 0.125 & $0.2223(2)$ & $0.61(2)$ & $0.63(2)$ & 0.91 & $0.5 \mathrm{k}$ \\
\hline 40 & 20 & 0.001 & 0.12 & $0.5099(5)$ & $1.10(2)$ & $1.10(2)$ & 0.9 & $1.5 \mathrm{k}$ \\
\hline 40 & 20 & 0.25 & 0.129 & $0.0168(1)$ & $0.48(2)$ & $0.50(3)$ & 0.88 & $1 \mathrm{k}$ \\
\hline 40 & 20 & 0.25 & 0.1281 & $0.0523(1)$ & $0.51(2)$ & $0.53(3)$ & 0.97 & $1.4 \mathrm{k}$ \\
\hline 40 & 20 & 0.25 & 0.1267 & $0.1032(1)$ & $0.54(3)$ & $0.57(3)$ & 0.97 & $0.8 \mathrm{k}$ \\
\hline 40 & 20 & 0.25 & 0.125 & $0.1700(2)$ & $0.55(2)$ & $0.55(2)$ & 0.92 & $0.8 \mathrm{k}$ \\
\hline 40 & 20 & 0.25 & 0.12 & $0.3823(2)$ & $0.801(2)$ & $0.801(2)$ & 0.91 & $2 \mathrm{k}$ \\
\hline 40 & 20 & 0.25 & 0.115 & $0.7534(6)$ & $1.373(7)$ & $1.374(7)$ & 0.92 & $2 \mathrm{k}$ \\
\hline
\end{tabular}

TABLE V. Same as Table II for $N_{t}=40, N_{s}=30$.

\begin{tabular}{|c|c|c|c|c|c|c|c|c|}
\hline$N_{t}$ & $N_{s}$ & $\beta_{L}$ & $\kappa$ & $m_{q}$ & $m_{\pi}$ & $m_{\rho}$ & acceptance rate & statistics \\
\hline 40 & 30 & -0.25 & 0.1309 & $0.0209(1)$ & $0.32(2)$ & $0.28(2)$ & 0.91 & $1.9 \mathrm{k}$ \\
\hline 40 & 30 & -0.25 & 0.1302 & $0.0489(1)$ & $0.40(2)$ & $0.39(2)$ & 0.92 & $0.8 \mathrm{k}$ \\
\hline 40 & 30 & -0.25 & 0.129 & $0.1015(2)$ & $0.39(3)$ & $0.38(3)$ & 0.75 & $0.3 \mathrm{k}$ \\
\hline 40 & 30 & -0.25 & 0.1277 & $0.1615(2)$ & $0.401(3)$ & $0.396(3)$ & 0.74 & $0.2 \mathrm{k}$ \\
\hline 40 & 30 & -0.25 & 0.1263 & $0.2270(2)$ & $0.535(8)$ & $0.535(8)$ & 0.77 & $1 \mathrm{k}$ \\
\hline 40 & 30 & -0.25 & 0.125 & $0.3018(2)$ & $0.670(1)$ & $0.670(1)$ & 0.79 & $2 \mathrm{k}$ \\
\hline 40 & 30 & -0.25 & 0.123 & $0.4557(6)$ & $0.981(6)$ & $0.981(6)$ & 0.76 & $2 \mathrm{k}$ \\
\hline 40 & 30 & 0.001 & 0.1299 & $0.0176(1)$ & $0.37(2)$ & $0.38(2)$ & 0.94 & $1.7 \mathrm{k}$ \\
\hline 40 & 30 & 0.001 & 0.129 & $0.0523(1)$ & $0.32(2)$ & $0.29(2)$ & 0.79 & $1 \mathrm{k}$ \\
\hline 40 & 30 & 0.001 & 0.1278 & $0.0991(1)$ & $0.40(2)$ & $0.38(2)$ & 0.94 & $1 \mathrm{k}$ \\
\hline
\end{tabular}


TABLE V. (Continued)

\begin{tabular}{|c|c|c|c|c|c|c|c|c|}
\hline$N_{t}$ & $N_{s}$ & $\beta_{L}$ & $\kappa$ & $m_{q}$ & $m_{\pi}$ & $m_{\rho}$ & acceptance rate & statistics \\
\hline 40 & 30 & 0.001 & 0.125 & $0.2185(2)$ & $0.514(3)$ & $0.509(3)$ & 0.92 & $0.5 \mathrm{k}$ \\
\hline 40 & 30 & 0.001 & 0.12 & $0.5091(4)$ & $1.040(5)$ & $1.040(5)$ & 0.8 & $2 \mathrm{k}$ \\
\hline 40 & 30 & 0.25 & 0.129 & $0.0155(1)$ & $0.29(2)$ & $0.25(2)$ & 0.81 & $1 \mathrm{k}$ \\
\hline 40 & 30 & 0.25 & 0.1281 & $0.0503(1)$ & $0.36(2)$ & $0.34(3)$ & 0.94 & $0.9 \mathrm{k}$ \\
\hline 40 & 30 & 0.25 & 0.1267 & $0.0994(1)$ & $0.29(1)$ & $0.27(1)$ & 0.94 & $0.5 \mathrm{k}$ \\
\hline 40 & 30 & 0.25 & 0.125 & $0.1659(2)$ & $0.393(2)$ & $0.387(2)$ & 0.81 & $0.4 \mathrm{k}$ \\
\hline 40 & 30 & 0.25 & 0.115 & $0.7552(8)$ & $1.398(6)$ & $1.398(6)$ & 0.83 & $2 \mathrm{k}$ \\
\hline
\end{tabular}

TABLE VI. Same as Table II for $N_{t}=40, N_{s}=40$.

\begin{tabular}{|c|c|c|c|c|c|c|c|c|}
\hline$N_{t}$ & $N_{s}$ & $\beta_{L}$ & $\kappa$ & $m_{q}$ & $m_{\pi}$ & $m_{\rho}$ & acceptance rate & statistics \\
\hline 40 & 40 & -0.25 & 0.1309 & $0.0203(1)$ & $0.25(3)$ & $0.22(3)$ & 0.91 & $0.9 \mathrm{k}$ \\
\hline 40 & 40 & -0.25 & 0.129 & $0.1005(1)$ & $0.262(1)$ & $0.255(1)$ & 0.88 & $0.8 \mathrm{k}$ \\
\hline 40 & 40 & -0.25 & 0.1277 & $0.1610(1)$ & $0.375(1)$ & $0.374(1)$ & 0.88 & $2 \mathrm{k}$ \\
\hline 40 & 40 & -0.25 & 0.1263 & $0.2272(1)$ & $0.523(2)$ & $0.521(2)$ & 0.9 & $2 \mathrm{k}$ \\
\hline 40 & 40 & -0.25 & 0.125 & $0.3023(2)$ & $0.672(1)$ & $0.672(1)$ & 0.89 & $2 \mathrm{k}$ \\
\hline 40 & 40 & -0.25 & 0.123 & $0.4561(5)$ & $0.974(2)$ & $0.975(2)$ & 0.88 & $2 \mathrm{k}$ \\
\hline 40 & 40 & 0.001 & 0.1299 & $0.0173(1)$ & $0.25(2)$ & $0.21(3)$ & 0.9 & $0.8 \mathrm{k}$ \\
\hline 40 & 40 & 0.001 & 0.129 & $0.0521(1)$ & $0.25(2)$ & $0.23(2)$ & 0.89 & $1.1 \mathrm{k}$ \\
\hline 40 & 40 & 0.001 & 0.125 & $0.2184(1)$ & $0.492(1)$ & $0.491(1)$ & 0.9 & $2 \mathrm{k}$ \\
\hline 40 & 40 & 0.001 & 0.12 & $0.5100(4)$ & $1.046(5)$ & $1.045(5)$ & 0.9 & $2 \mathrm{k}$ \\
\hline 40 & 40 & 0.25 & 0.129 & $0.0151(1)$ & $0.20(2)$ & $0.17(2)$ & 0.91 & $0.9 \mathrm{k}$ \\
\hline 40 & 40 & 0.25 & 0.125 & $0.1662(1)$ & $0.377(2)$ & $0.376(2)$ & 0.91 & $2 \mathrm{k}$ \\
\hline 40 & 40 & 0.25 & 0.12 & $0.3868(2)$ & $0.807(2)$ & $0.807(2)$ & 0.92 & $2 \mathrm{k}$ \\
\hline 40 & 40 & 0.25 & 0.115 & $0.758(2)$ & $1.41(1)$ & $1.42(1)$ & 0.92 & $2 \mathrm{k}$ \\
\hline
\end{tabular}

TABLE VII. $\quad$ Same as Table II for $N_{t}=48, N_{s}=24$.

\begin{tabular}{|c|c|c|c|c|c|c|c|c|}
\hline$N_{t}$ & $N_{s}$ & $\beta_{L}$ & $\kappa$ & $m_{q}$ & $m_{\pi}$ & $m_{\rho}$ & acceptance rate & statistics \\
\hline 48 & 24 & -0.25 & 0.1309 & $0.0214(1)$ & $0.44(2)$ & $0.45(2)$ & 0.94 & $2 \mathrm{k}$ \\
\hline 48 & 24 & -0.25 & 0.1302 & $0.0497(1)$ & $0.43(2)$ & $0.40(2)$ & 0.95 & $1.6 \mathrm{k}$ \\
\hline 48 & 24 & -0.25 & 0.129 & $0.1027(2)$ & $0.41(2)$ & $0.43(3)$ & 0.82 & $2 \mathrm{k}$ \\
\hline 48 & 24 & -0.25 & 0.1277 & $0.1608(1)$ & $0.435(7)$ & $0.443(7)$ & 0.81 & $2 \mathrm{k}$ \\
\hline 48 & 24 & -0.25 & 0.1263 & $0.2245(1)$ & $0.515(1)$ & $0.516(2)$ & 0.81 & $2 \mathrm{k}$ \\
\hline 48 & 24 & -0.25 & 0.125 & $0.2991(3)$ & $0.674(3)$ & $0.674(3)$ & 0.83 & $2 \mathrm{k}$ \\
\hline 48 & 24 & -0.25 & 0.123 & $0.4539(4)$ & $0.990(8)$ & $0.990(9)$ & 0.82 & $2 \mathrm{k}$ \\
\hline 48 & 24 & 0.001 & 0.1299 & $0.0182(1)$ & $0.41(2)$ & $0.42(2)$ & 0.95 & $2 \mathrm{k}$ \\
\hline 48 & 24 & 0.001 & 0.129 & $0.0535(2)$ & $0.45(2)$ & $0.41(2)$ & 0.84 & $2 \mathrm{k}$ \\
\hline 48 & 24 & 0.001 & 0.1278 & $0.1003(1)$ & $0.49(1)$ & $0.47(2)$ & 0.95 & $1.8 \mathrm{k}$ \\
\hline 48 & 24 & 0.001 & 0.125 & $0.2168(2)$ & $0.508(2)$ & $0.505(2)$ & 0.84 & $2 \mathrm{k}$ \\
\hline 48 & 24 & 0.001 & 0.12 & $0.5063(4)$ & $1.037(5)$ & $1.037(5)$ & 0.85 & $2 \mathrm{k}$ \\
\hline 48 & 24 & 0.25 & 0.129 & $0.0161(1)$ & $0.42(2)$ & $0.43(3)$ & 0.86 & $2 \mathrm{k}$ \\
\hline 48 & 24 & 0.25 & 0.1281 & $0.0510(1)$ & $0.42(2)$ & $0.39(2)$ & 0.95 & $1.8 \mathrm{k}$ \\
\hline 48 & 24 & 0.25 & 0.1267 & $0.1008(2)$ & $0.385(7)$ & $0.362(7)$ & 0.95 & $1.8 \mathrm{k}$ \\
\hline 48 & 24 & 0.25 & 0.125 & $0.1648(1)$ & $0.382(2)$ & $0.384(2)$ & 0.86 & $2 \mathrm{k}$ \\
\hline 48 & 24 & 0.25 & 0.12 & $0.3821(2)$ & $0.798(1)$ & $0.798(1)$ & 0.87 & $2 \mathrm{k}$ \\
\hline 48 & 24 & 0.25 & 0.115 & $0.7508(8)$ & $1.384(8)$ & $1.385(8)$ & 0.89 & $2 \mathrm{k}$ \\
\hline
\end{tabular}


TABLE VIII. Same as Table II for $N_{t}=48, N_{s}=36$.

\begin{tabular}{|c|c|c|c|c|c|c|c|c|}
\hline$N_{t}$ & $N_{s}$ & $\beta_{L}$ & $\kappa$ & $m_{q}$ & $m_{\pi}$ & $m_{\rho}$ & acceptance rate & statistics \\
\hline 48 & 36 & -0.25 & 0.1309 & $0.0205(1)$ & $0.28(2)$ & $0.25(3)$ & 0.88 & $1 \mathrm{k}$ \\
\hline 48 & 36 & -0.25 & 0.129 & $0.0999(1)$ & $0.238(1)$ & $0.237(1)$ & 0.89 & $2 \mathrm{k}$ \\
\hline 48 & 36 & -0.25 & 0.1277 & $0.1602(1)$ & $0.372(1)$ & $0.372(1)$ & 0.9 & $2 \mathrm{k}$ \\
\hline 48 & 36 & -0.25 & 0.1263 & $0.2260(1)$ & $0.513(1)$ & $0.513(1)$ & 0.88 & $2 \mathrm{k}$ \\
\hline 48 & 36 & -0.25 & 0.125 & $0.3006(2)$ & $0.670(2)$ & $0.670(2)$ & 0.89 & $2 \mathrm{k}$ \\
\hline 48 & 36 & -0.25 & 0.123 & $0.4538(5)$ & $0.973(3)$ & $0.973(3)$ & 0.89 & $2 \mathrm{k}$ \\
\hline 48 & 36 & 0.001 & 0.1299 & $0.0173(1)$ & $0.30(3)$ & $0.28(3)$ & 0.9 & $2.4 \mathrm{k}$ \\
\hline 48 & 36 & 0.001 & 0.129 & $0.0520(1)$ & $0.24(2)$ & $0.22(2)$ & 0.91 & $0.6 \mathrm{k}$ \\
\hline 48 & 36 & 0.001 & 0.125 & $0.2175(1)$ & $0.488(1)$ & $0.487(1)$ & 0.91 & $2 \mathrm{k}$ \\
\hline 48 & 36 & 0.001 & 0.12 & $0.5073(3)$ & $1.038(3)$ & $1.039(3)$ & 0.91 & $2 \mathrm{k}$ \\
\hline 48 & 36 & 0.25 & 0.129 & $0.0152(1)$ & $0.25(2)$ & $0.21(2)$ & 0.92 & $1.6 \mathrm{k}$ \\
\hline 48 & 36 & 0.25 & 0.125 & $0.1653(1)$ & $0.376(1)$ & $0.375(1)$ & 0.91 & $2 \mathrm{k}$ \\
\hline 48 & 36 & 0.25 & 0.12 & $0.3851(2)$ & $0.803(1)$ & $0.803(1)$ & 0.92 & $2 \mathrm{k}$ \\
\hline 48 & 36 & 0.25 & 0.115 & $0.754(1)$ & $1.42(2)$ & $1.42(2)$ & 0.92 & $2 \mathrm{k}$ \\
\hline
\end{tabular}

TABLE IX. Same as Table II for $N_{t}=48, N_{s}=48$.

\begin{tabular}{|c|c|c|c|c|c|c|c|c|}
\hline$N_{t}$ & $N_{s}$ & $\beta_{L}$ & $\kappa$ & $m_{q}$ & $m_{\pi}$ & $m_{\rho}$ & acceptance rate & statistics \\
\hline 48 & 48 & -0.25 & 0.1309 & $0.0202(1)$ & $0.16(2)$ & $0.13(3)$ & 0.92 & $1.4 \mathrm{k}$ \\
\hline 48 & 48 & -0.25 & 0.129 & $0.1001(1)$ & $0.239(1)$ & $0.238(1)$ & 0.91 & $2.9 \mathrm{k}$ \\
\hline 48 & 48 & -0.25 & 0.1277 & $0.1608(1)$ & $0.373(1)$ & $0.372(1)$ & 0.9 & $3 \mathrm{k}$ \\
\hline 48 & 48 & -0.25 & 0.1263 & $0.2266(1)$ & $0.516(1)$ & $0.516(1)$ & 0.91 & $3.2 \mathrm{k}$ \\
\hline 48 & 48 & -0.25 & 0.125 & $0.3013(2)$ & $0.672(1)$ & $0.672(1)$ & 0.91 & $3.3 \mathrm{k}$ \\
\hline 48 & 48 & -0.25 & 0.123 & $0.4546(3)$ & $0.976(2)$ & $0.976(2)$ & 0.91 & $3.5 \mathrm{k}$ \\
\hline 48 & 48 & 0.001 & 0.1299 & $0.0170(1)$ & $0.19(2)$ & $0.16(3)$ & 0.92 & $2.1 \mathrm{k}$ \\
\hline 48 & 48 & 0.001 & 0.129 & $0.0517(1)$ & $0.173(4)$ & $0.158(4)$ & 0.93 & $1.3 \mathrm{k}$ \\
\hline 48 & 48 & 0.001 & 0.125 & $0.2179(1)$ & $0.492(1)$ & $0.491(1)$ & 0.92 & $3.2 \mathrm{k}$ \\
\hline 48 & 48 & 0.001 & 0.12 & $0.5074(3)$ & $1.052(3)$ & $1.053(3)$ & 0.93 & $3.6 \mathrm{k}$ \\
\hline 48 & 48 & 0.25 & 0.129 & $0.0151(1)$ & $0.18(3)$ & $0.15(3)$ & 0.91 & $1.9 \mathrm{k}$ \\
\hline 48 & 48 & 0.25 & 0.125 & $0.1658(1)$ & $0.373(1)$ & $0.373(1)$ & 0.93 & $2.9 \mathrm{k}$ \\
\hline 48 & 48 & 0.25 & 0.12 & $0.3853(1)$ & $0.810(3)$ & $0.810(3)$ & 0.93 & $3.6 \mathrm{k}$ \\
\hline 48 & 48 & 0.25 & 0.115 & $0.7534(7)$ & $1.42(2)$ & $1.42(2)$ & 0.94 & $3.4 \mathrm{k}$ \\
\hline
\end{tabular}

[1] F. Sannino and K. Tuominen, Orientifold theory dynamics and symmetry breaking, Phys. Rev. D 71, 051901 (2005).

[2] C. T. Hill and E. H. Simmons, Strong dynamics and electroweak symmetry breaking, Phys. Rep. 381, 235 (2003); 390, 553(E) (2004).

[3] D. D. Dietrich, F. Sannino, and K. Tuominen, Light composite Higgs from higher representations versus electroweak precision measurements: Predictions for CERN LHC, Phys. Rev. D 72, 055001 (2005).

[4] A. Arbey, G. Cacciapaglia, H. Cai, A. Deandrea, S. Le Corre, and F. Sannino, Fundamental Composite Electroweak Dynamics: Status at the LHC, Phys. Rev. D 95, 015028 (2017).
[5] T. Karavirta, J. Rantaharju, K. Rummukainen, and K. Tuominen, Determining the conformal window: SU(2) gauge theory with $N_{f}=4,6$ and 10 fermion flavours, J. High Energy Phys. 05 (2012) 003.

[6] V. Leino, J. Rantaharju, T. Rantalaiho, K. Rummukainen, J. M. Suorsa, and K. Tuominen, The gradient flow running coupling in $\mathrm{SU}(2)$ gauge theory with $N_{f}=8$ fundamental flavors, Phys. Rev. D 95, 114516 (2017).

[7] V. Leino, K. Rummukainen, J. M. Suorsa, K. Tuominen, and S. Tähtinen, Infrared fixed point of SU(2) gauge theory with six flavors, Phys. Rev. D 97, 114501 (2018).

[8] V. Leino, K. Rummukainen, and K. Tuominen, Slope of the beta function at the fixed point of $\mathrm{SU}(2)$ gauge theory with six or eight flavors, Phys. Rev. D 98, 054503 (2018). 
[9] A. Amato, V. Leino, K. Rummukainen, K. Tuominen, and S. Tähtinen, From chiral symmetry breaking to conformality in SU(2) gauge theory, arXiv:1806.07154.

[10] A. J. Hietanen, J. Rantaharju, K. Rummukainen, and K. Tuominen, Spectrum of SU(2) lattice gauge theory with two adjoint Dirac flavours, J. High Energy Phys. 05 (2009) 025 .

[11] A. J. Hietanen, K. Rummukainen, and K. Tuominen, Evolution of the coupling constant in SU(2) lattice gauge theory with two adjoint fermions, Phys. Rev. D 80, 094504 (2009).

[12] L. Del Debbio, A. Patella, and C. Pica, Higher representations on the lattice: Numerical simulations. SU(2) with adjoint fermions, Phys. Rev. D 81, 094503 (2010).

[13] L. Del Debbio, B. Lucini, A. Patella, C. Pica, and A. Rago, Conformal versus confining scenario in SU(2) with adjoint fermions, Phys. Rev. D 80, 074507 (2009).

[14] L. Del Debbio, B. Lucini, A. Patella, C. Pica, and A. Rago, Mesonic spectroscopy of Minimal Walking Technicolor, Phys. Rev. D 82, 014509 (2010).

[15] F. Bursa, L. Del Debbio, D. Henty, E. Kerrane, B. Lucini, A. Patella, C. Pica, T. Pickup, and A. Rago, Improved lattice spectroscopy of minimal walking technicolor, Phys. Rev. D 84, 034506 (2011).

[16] T. DeGrand, Y. Shamir, and B. Svetitsky, Infrared fixed point in SU(2) gauge theory with adjoint fermions, Phys. Rev. D 83, 074507 (2011).

[17] J. Rantaharju, T. Rantalaiho, K. Rummukainen, and K. Tuominen, Running coupling in SU(2) gauge theory with two adjoint fermions, Phys. Rev. D 93, 094509 (2016).

[18] L. Del Debbio, B. Lucini, A. Patella, C. Pica, and A. Rago, Large volumes and spectroscopy of walking theories, Phys. Rev. D 93, 054505 (2016).

[19] Y. Aoki et al. (LatKMI Collaboration), Walking signals in $N_{f}=8$ QCD on the lattice, Phys. Rev. D 87, 094511 (2013).

[20] Y. Aoki et al. (LatKMI Collaboration), Light Composite Scalar in Twelve-Flavor QCD on the Lattice, Phys. Rev. Lett. 111, 162001 (2013).

[21] Z. Fodor, K. Holland, J. Kuti, D. Nogradi, C. Schroeder, K. Holland, J. Kuti, D. Nogradi, and C. Schroeder, Twelve massless flavors and three colors below the conformal window, Phys. Lett. B 703, 348 (2011).

[22] A. Hasenfratz, D. Schaich, and A. Veernala, Nonperturbative $\beta$ function of eight-flavor SU(3) gauge theory, J. High Energy Phys. 06 (2015) 143.

[23] Z. Fodor, K. Holland, J. Kuti, S. Mondal, D. Nogradi, and C. H. Wong, The running coupling of 8 flavors and 3 colors, J. High Energy Phys. 06 (2015) 019.

[24] Z. Fodor, K. Holland, J. Kuti, D. Nogradi, and C. H. Wong, Extended investigation of the twelve-flavor $\beta$-function, Phys. Lett. B 779, 230 (2018).

[25] T. Appelquist et al. (Lattice Strong Dynamics Collaboration), Nonperturbative investigations of SU(3) gauge theory with eight dynamical flavors, Phys. Rev. D 99, 014509 (2019).
[26] A. Hasenfratz, C. Rebbi, and O. Witzel, Gradient flow stepscaling function for SU(3) with twelve flavors, Phys. Rev. D 100, 114508 (2019).

[27] A. Hasenfratz, C. Rebbi, and O. Witzel, Gradient flow stepscaling function for $\mathrm{SU}(3)$ with ten fundamental flavors, Phys. Rev. D 101, 114508 (2020).

[28] T. Appelquist et al. (Lattice Strong Dynamics Collaboration), Near-conformal dynamics in a chirally broken system, Phys. Rev. D 103, 014504 (2021).

[29] Y. Shamir, B. Svetitsky, and T. DeGrand, Zero of the discrete beta function in SU(3) lattice gauge theory with color sextet fermions, Phys. Rev. D 78, 031502 (2008).

[30] T. DeGrand, Y. Shamir, and B. Svetitsky, Phase structure of SU(3) gauge theory with two flavors of symmetricrepresentation fermions, Phys. Rev. D 79, 034501 (2009).

[31] Z. Fodor, K. Holland, J. Kuti, D. Nogradi, and C. Schroeder, Chiral properties of SU(3) sextet fermions, J. High Energy Phys. 11 (2009) 103.

[32] T. DeGrand, Y. Shamir, and B. Svetitsky, Running coupling and mass anomalous dimension of SU(3) gauge theory with two flavors of symmetric-representation fermions, Phys. Rev. D 82, 054503 (2010).

[33] Z. Fodor, K. Holland, J. Kuti, S. Mondal, D. Nogradi, and C. H. Wong, The running coupling of the minimal sextet composite Higgs model, J. High Energy Phys. 09 (2015) 039.

[34] T. DeGrand, Y. Shamir, and B. Svetitsky, SU(4) lattice gauge theory with decuplet fermions: Schrödinger functional analysis, Phys. Rev. D 85, 074506 (2012).

[35] T. DeGrand, Y. Liu, E. T. Neil, Y. Shamir, and B. Svetitsky, Spectroscopy of SU(4) gauge theory with two flavors of sextet fermions, Phys. Rev. D 91, 114502 (2015).

[36] V. Leino, T. Rindlisbacher, K. Rummukainen, F. Sannino, and K. Tuominen, Safety versus triviality on the lattice, Phys. Rev. D 101, 074508 (2020).

[37] F. Jegerlehner and O. V. Tarasov, Exact mass dependent two loop anti-alpha(s) $\left(\mathrm{Q}^{* * 2}\right)$ in the background MOM renormalization scheme, Nucl. Phys. B549, 481 (1999).

[38] S. Capitani, S. Durr, and C. Hoelbling, Rationale for UVfiltered clover fermions, J. High Energy Phys. 11 (2006) 028.

[39] R. C. Brower, T. Ivanenko, A. R. Levi, and K. N. Orginos, Chronological inversion method for the Dirac matrix in hybrid Monte Carlo, Nucl. Phys. B484, 353 (1997).

[40] M. Lüscher and P. Weisz, O(a) improvement of the axial current in lattice QCD to one loop order of perturbation theory, Nucl. Phys. B479, 429 (1996).

[41] A. Hasenfratz and T. A. DeGrand, Heavy dynamical fermions in lattice QCD, Phys. Rev. D 49, 466 (1994).

[42] T. Blum, C. E. DeTar, U. M. Heller, L. Karkkainen, K. Rummukainen, and D. Toussaint, Thermal phase transition in mixed action SU(3) lattice gauge theory and Wilson fermion thermodynamics, Nucl. Phys. B442, 301 (1995).

[43] P. de Forcrand, S. Kim, and W. Unger, Conformality in many-flavour lattice QCD at strong coupling, J. High Energy Phys. 02 (2013) 051.

[44] M. Albanese et al. (APE Collaboration), Glueball masses and string tension in lattice QCD, Phys. Lett. B 192, 163 (1987). 
[45] B. Bolder, T. Struckmann, G. S. Bali, N. Eicker, T. Lippert, B. Orth, K. Schilling, and P. Ueberholz, A High precision study of the Q anti-Q potential from Wilson loops in the regime of string breaking, Phys. Rev. D 63, 074504 (2001).
[46] M. Teper, An improved method for lattice glueball calculations, Phys. Lett. B 183, 345 (1987).

[47] B. Lucini, A. Rago, and E. Rinaldi, Glueball masses in the large N limit, J. High Energy Phys. 08 (2010) 119. 